\title{
ELECTROCARDIOGRAPHIC CHANGES DUE TO TRAUMA
}

\author{
BY \\ HUGH BARBER \\ From the Derbyshire Royal Infirmary \\ Received April 25, 1942
}

Many electrocardiograms have been recorded in cases of trauma of the heart. Beck (1935 and 1940) has studied the problem in the surgical wards and in animal experiments. Kissane (1937 and 1940) has published clinical and experimental records, and in Warburg's analysis of cases (1938 and 1940) there are his own tracings and those of other workers. Anderson (1940) published a case, with full clinical details and convincing electrocardiographic proof of myocardial injury. Zuckerman (1942) and Kessler (1941) record experiments.

From this hospital (Barber, 1938 and 1940) there are clinical notes of case histories suggesting heart trauma dating back to 1910; and there is evidence from accident autopsies (Barber and Osborn, 1941).

Both animal experiments and clinical records suggest that the electrocardiogram should be taken quite soon after the injury, because abnormalities may be in evidence only for a short time.

This paper gives an account of a series of electrocardiograms from patients, irrespective of their symptoms, who came to hospital as the direct result of a severe blow over the front of the chest, or some crushing injury of the thorax. Most of the examinations were made within a few hours of the accident and all within 48 hours; the patients being seen in the casualty department, the radiological department, or a surgical ward. The total number examined was thirty-three, in whom there were eight abnormal tracings of definite but varying significance, and two that were inconclusive.

The accidents were all from traffic or industry; not one of them was the result of enemy action. They were selected, as opportunity arose, on the basis of having sustained a reasonably severe blow or injury. All those showing an abnormal tracing were with one exception below 45 years in age, and they were active people free from degenerative processes. The youngest, aged 17 years, who developed heart block from a crushing injury of the thorax, is a good illustration of the fact that in the young and elastic chest the heart may be injured without fractures of bone.

There was rather a high proportion of seriously injured in the series, but that is to be expected, if one seeks material coming to hospital soon after 
accident. There is ample evidence, in the reported cases, of heart trauma after accidents that had seemed to be comparatively trivial. One need only look at the cardiographic pictures to see that an early tracing is required.

No two accidents are quite alike, but comparison with the experimental work in animals will show that many of the abnormal tracings recorded therein have been met with in this series of hospital accident cases. Analysis of the symptoms is not profitable with a series so small and variable. Moreover, early symptoms of a cardiac lesion may have been masked by the associated injuries, and in some cases later ones kept in check by the necessary rest in bed. Some of the most convincing clinical histories suggesting myocardial contusion recount a blow or crushing injury; then an interval, variable in time, before symptoms of dyspnœa and angina develop; and perhaps no heart investigation until two or three weeks later. It is in this way that proof may be unobtainable. And by way of contrast, one or two cases in this series, with significant temporary changes in the $T$ waves, may have shown little or no evidence from symptoms, because the associated chest injuries necessitated a rest in bed until the heart had recovered.

From the publications referred to, it is possible to find complete clinical pictures of traumatic heart lesions with history, symptoms, and signs; but this paper is confined to an analysis of the electrocardiograms in a series of accidents. There was no example of auricular fibrillation, nor was there a picture resembling coronary thrombosis. There was one example of partial heart block. These three abnormalities are the most likely to be associated with clinical symptoms of long duration in a case of trauma.

The abnormal tracings in this series have been one example of partial heart block, one of sinus bradycardia, one of slurring of the $R$ waves, and five with temporary changes in the $T$ waves. Two more with changes in lead III alone are considered doubtful. In view of the recent publication of White, Chamberlain, and Graybiel (1941) that the T wave in lead II may sometimes vary with a change in position of the patient, it is necessary to say that all the tracings were taken with the patient lying down. Brief notes of the cases follow.

\section{CASE Notes}

Case 1. Partial Heart Block (Fig. 1). C. M., male, aged 17 years. On November 11,1941 , at 8.30 a.m., he was crushed between the radiator of a large lorry and a wall. He was doubled up with pain for a few minutes and coughed up a little bloodstained material. He soon recovered and cycled up to hospital. Examined at 10 a.m. there was no distress, the X-ray evidence was negative, except for slight dimness in the left lung (which passed off in two days): the pulse rate was 45 to 50 , not quite regular, with a tendency to have three beats and a pause; the first sound was prolonged; there was also an occasional premature ventricular contraction. The cardiogram showed partial heart block (see Fig. 1). The blood pressure was 115 systolic and 55 diastolic. He was detained in hospital three weeks with no change in his condition or his tracing. The heart rate increased to 68 to 72 . A month after the accident, he was short of breath if he walked more than a mile. Three months after he had no discomfort unless he ran, and he had started light work. Numerous tracings had shown no change in the long $\mathrm{P}-\mathrm{R}$ interval. After five months the heart rate was 
72, the blood pressure was 120 systolic and 80 diastolic, and the tracing unchanged, except that the P-R interval was more uniform in length, 0.35 of a second. He was working successfully but not capable of running without discomfort. He had not

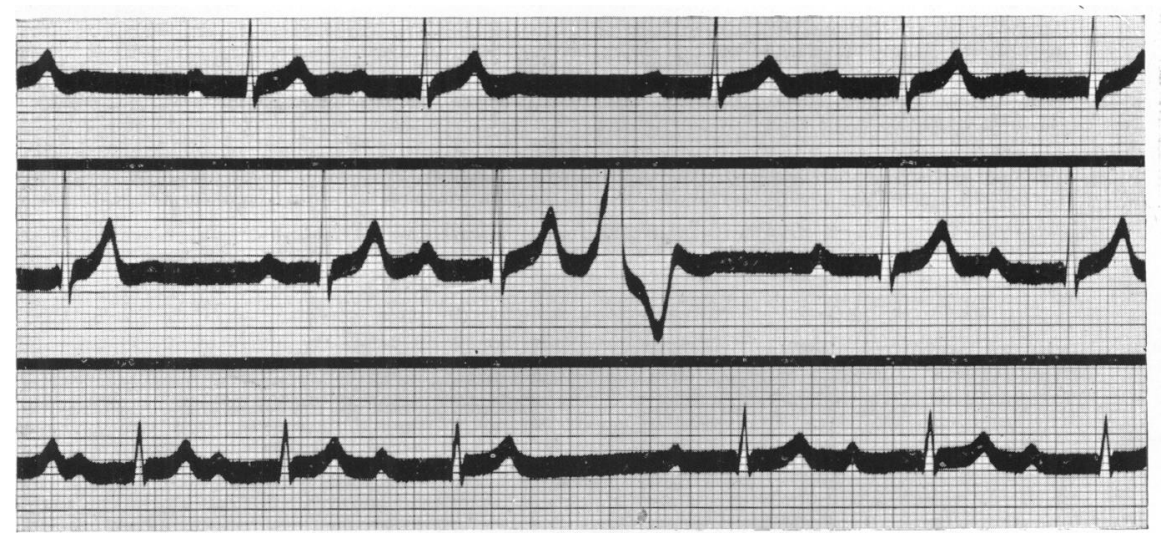

FIG. 1.-Partial heart block after trauma.

had rheumatic fever or scarlet fever, and the Wassermann was negative. There was no evidence from his doctor to suggest that he had heart disease from natural causes, and he was a vigorous cyclist and player of games before the accident.

Case 2. Sinus Bradycardia (Fig. 2). B. F., male, aged 33 years. On February 2, 1942, he was driving a trolley-bus, which skidded and ran into a wall. He bruised his chest on the steering-wheel and was "winded," but cleared up the accident; and an hour later drove off with another bus. After driving for a quarter of an hour

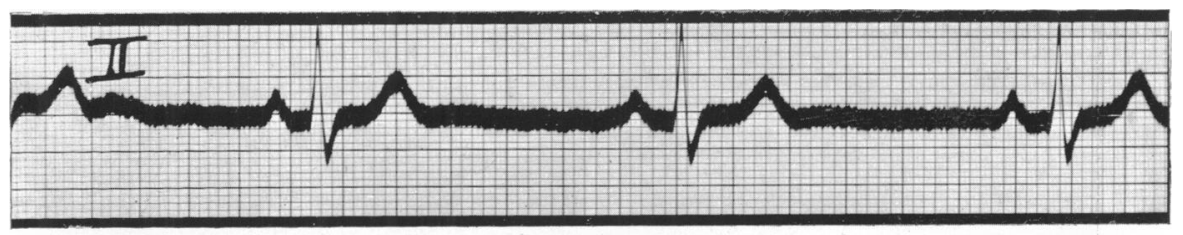

FIG. 2.-Sinus bradycardia after trauma.

he fainted, quite unexpectedly. He soon recovered and was brought to hospital. Nothing abnormal was found, except for a slow heart rate of 44 . He was admitted for two days; when the heart rate taken every hour varied between 36 and 46 . He reported on the fifth day, free from symptoms, with a heart rate of 45 and a normal tracing. On the twelfth day the rate was 72 and he was quite well. Sinus bradycardia has been recorded in animal experiments.

Case 3. Slurring of the $R$ waves (Fig. 3). F. C., male, aged 26 years. On January 18,1939 , he was struck on the front of the chest by a fall of limestone, which crushed him against a lorry. The left clavicle and the third, fourth, and fifth ribs were fractured and the left scapula. He was admitted to a surgical ward, rather shocked, with a pulse rate between 90 and 100 , but no other signs of heart lesion except slurring of the $\mathrm{R}$ waves in all three leads. On February 2 there was no change, but on February 7 it was only definite in lead I. He went home after six weeks and was at work, feeling quite well, three months after the accident. Asked to report three years after the accident, he gave a history of excellent health and his cardiogram was normal in all four leads. Slurring of the $\mathbf{R}$ waves has been recorded in animal experiments. 
I

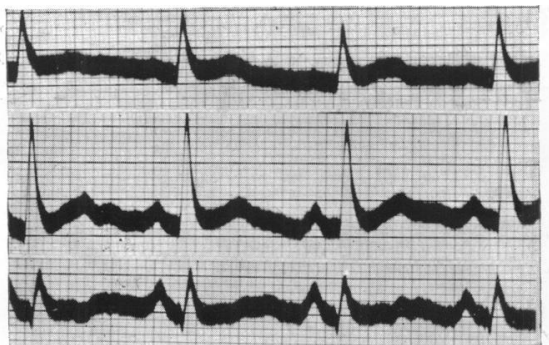

A

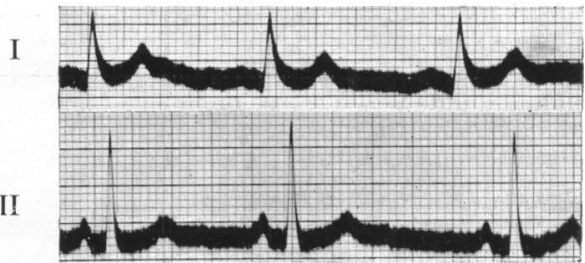

B

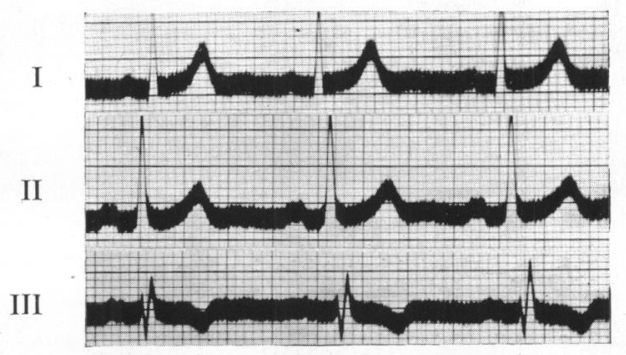

$\mathrm{C}$

FIG. 3.-Slurring of the $\mathrm{R}$ waves. (A) $18 / 1 / 39$. (B) $7 / 2 / 39$. (C) $5 / 3 / 42$.

Case 4. T waves of exaggerated amplitude (Fig. 4). G. W., male, aged 35 years. On January 20, 1942, he was kicked over the front of the chest by a horse, which knocked him across the stable. Seen in the Casualty Department an hour later, he

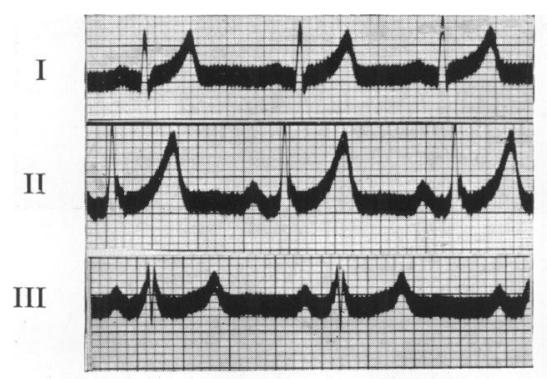

A

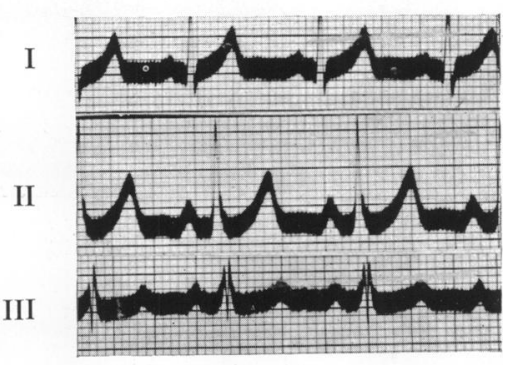

B

II

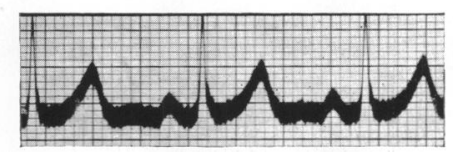

C

FIG. 4.- $\mathrm{T}$ waves of exaggerated amplitude after trauma. (A) 20/1/42. (B) $23 / 1 / 42$. (C) $27 / 1 / 42$.

was free from distress. The X-ray and other findings were normal, but the electrocardiogram showed $T$ waves of an amplitude almost equal to that of the $R$ waves. Three days later the proportions were normal; bruising was noticed around the costal 
cartilages between the nipple and the sternum. Seven days after the accident the tracing was normal. There had been no symptoms of discomfort apart from the bruising. Beck (1935) has recorded this finding.

Case 5. Temporary changes in $T$ waves (Fig. 5). E. D., female, aged 31 years. On December 3, 1941, she was thrown out of a motor car. There was bruising of the back and chest. Two ribs were fractured on the right side, also the right scapula and one rib on the left side. She was very distressed, with extensive lung contusion on

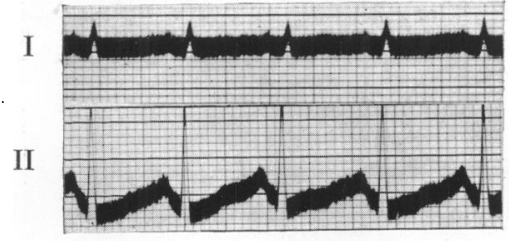

A

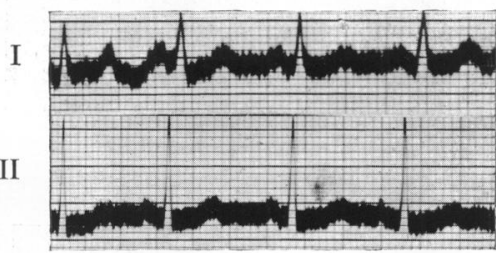

B

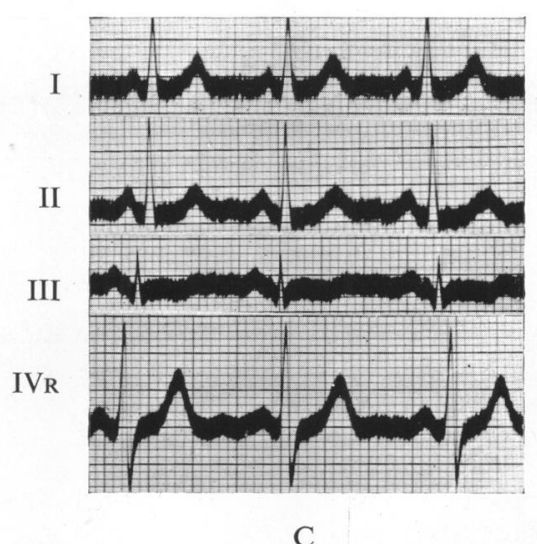

Fig. 5.-Temporary changes in $T_{1}$ and $T_{2}$ after trauma. (A) $4 / 12 / 41$. (B) $15 / 12 / 41$. (C) $6 / 1 / 42$.

both sides and pleurisy over the left lung. The T waves in leads I and II were flat or absent. On the sixth and seventh days there was a pericardial friction sound, but this was only definite on these two days. Twelve days after the accident the $T$ waves were upright, and after five weeks the tracing was normal in all four leads. In the fourth week, when she was well enough to screen properly, a small but definite pericardial effusion was recognizable. She travelled to her home by car thirty miles in the eighth week; and reported four months after the accident that she was getting about quite well but sometimes had a tightness in the chest if she hurried. The early return to normal of the $\mathrm{T}$ waves is an important observation, in view of the $\mathrm{X}$-ray evidence later.

Case 6. Temporary Changes in the T waves (Fig. 6). J. M., male, aged 28 years. On December 4, 1941, he was felling a tree, when the trunk swung round and crushed him against the stump. He was admitted with five ribs fractured on the right side and his left scapula; also fracture of the seventh dorsal vertebra. He was admitted rather shocked with a quick heart rate. Next day the electrocardiogram showed a flat $\mathrm{T}$ wave in lead II, which was little improved four days later, but was quite normal on the seventeenth day after the accident. He was up in a spinal jacket in two months, and was quite well in himself three months after the accident, having exercises for the spine. 


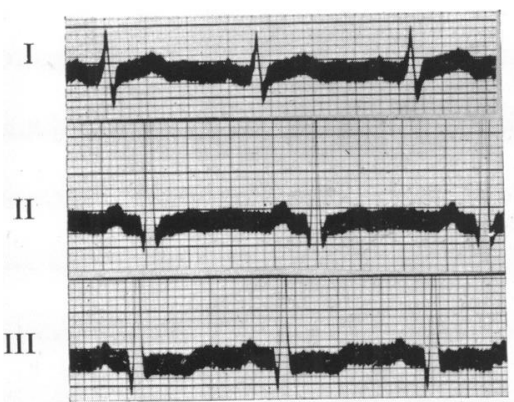

A

II

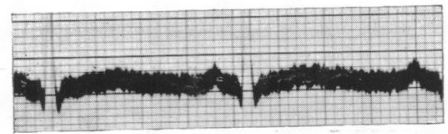

B

\section{I}

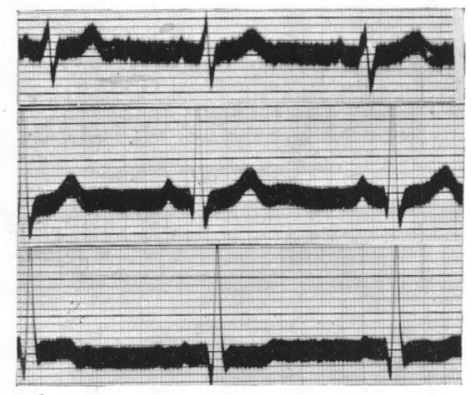

C

days later this was unchanged. He had a large heart with the diastolic blood pressure 110 and the systolic 180 . His arteries were thick and tortuous. He went home after two weeks, and subsequent tracings (Fig. 7) show the $\mathrm{T}$ wave improving until it was definitely upright eight weeks after the accident. In six months he was back at work again. 
Case 8. Temporary Changes in the $T$ waves (Fig. 8). F. M., male, aged 45 years. On March 3, 1942, he was crushed between two buffers. There was extensive bruising over the trunk, with subcutaneous emphysema over the left side. He was very shocked, with a heart rate of 100 and sometimes 140 . The second, third, and fourth ribs were

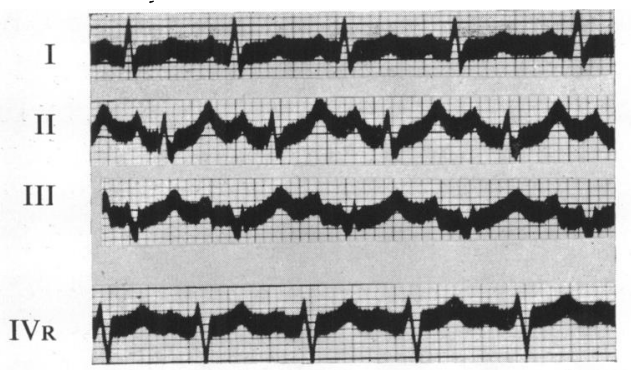

A
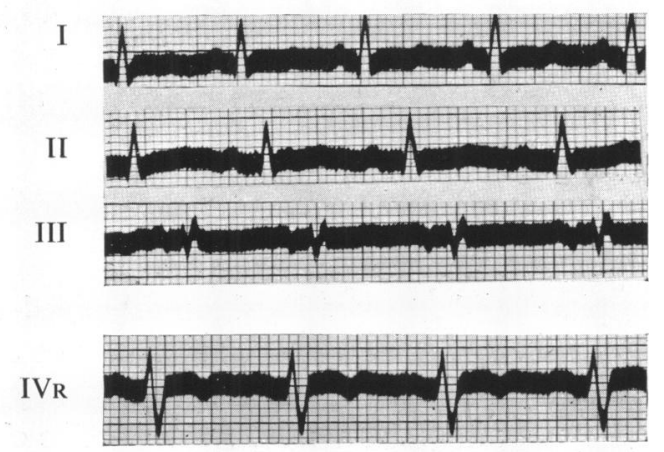

B

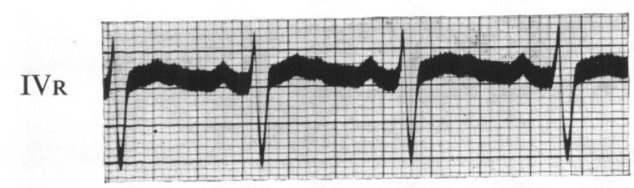

$\mathrm{C}$

Fig. 8.-Temporary changes in $T$ waves of all leads after trauma. (A) 10/3/42. (B) $13 / 3 / 42$. (C) $16 / 3 / 42$.

fractured on the left side and also the left scapula. The cardiogram a few hours after the accident was normal. Three days later his general condition was rather improved, but the tracing showed inversion of the T wave in lead IVR and the other $T$ waves were flat (Fig. 8). In another three days the $T$ waves were normal again. $\mathrm{He}$ progressed favourably and was getting about the ward by the end of five weeks.

Cases 9 and 10. Temporary Changes in T wave of lead III only. There were two examples of this, one in a man who had a large bruise over the sternum from a blow from a machine belt, and the other in a man who was crushed between two buffers. In neither case was there a fracture. Both were free from any serious symptoms.

As an explanation of this finding the possibility of a change in the height of the diaphragm must be considered. In one of them examinations could be made that appeared to exclude this, but the other was confined to bed. 


\section{ANALYSIS OF THE ACCIDENTS}

The accidents may be grouped rather loosely as follows:

1. Crushed by a fall of roof in a coal mine: four cases, illustrated by Fig. 7.

2. Crushed between two buffers, or similar accident: seven cases, illustrated by Fig. 1, 6, and 8 .

3. Chest injuries against steering-wheel of car: one case, illustrated by Fig. 2.

4. A severe fall on to the chest: six cases, illustrated by Fig. 5.

5. Kicked on the chest by a horse: two cases, illustrated by Fig. 4.

6. A severe blow over the chest or left side: thirteen cases, illustrated by Fig. 3 ; in this group were the two doubtful tracings, 9 and 10, not illustrated.

It is of interest that there should be only one "steering-wheel" accident, because it is a fairly common one, of which there have been two more examples at this hospital recently. They are excluded from this series, because they did not come to hospital until the fourth week after the accident. Both had a crack in the sternum and were complaining of anginal symptoms, but the electrocardiogram was normal at this stage. One, aged 40 years, recovered completely after about six weeks, but the other, aged 51 years, complains of some limitations to his response to exertion six months after the accident. He has no claim to compensation.

\section{SUMMARY}

A series of 33 hospital accident cases has been examined within two days of the injury. Patients with a severe blow over the chest or a crushing injury of the thorax were selected as opportunity arose.

Eight abnormal electrocardiograms were obtained. The changes observed are comparable with results obtained in animal experiments.

My thanks are due to my surgical colleagues and to Mr. G. Lovell Stiles for his help in the X-ray department and with the tracings.

\section{REFERENCES}

Anderson, R. G. (1940). Brit. med. J., 2, 307.

Barber, H. (1938). Ibid., 1, 433. (1940). Ibid., 2, 520.

and Osborn, G. R. (1941). Brit. Heart J., 3, 127.

Beck, C. S. (1935). J. Amer. med. Ass., 104, 109.

- (1940). Diagnosis and Treatment of Cardiovascular Disease. Edited by Stroud, Philadelphia, vol. 2, p. 1157.

Bright, E. F., and Beck, C. S. (1935). Amer. Heart J., 10, 293.

Kessler, H. H. (1941). Accidental Injuries. New Jersey, p. 387.

Kissane, R. W. (1937). Contusion of the Heart. Ohio State University, Columbus.

-, Fidler, R. S., and Koons, R. A. (1937). Ann. intern. Med., 11, 907.

- (1940). Advancement of Science, 13, 170.

Warburg, E. (1938). Subacute and Chronic Pericardial and Myocardial Lesions due to NonPenetrating Traumatic Injuries. Copenhagen.

- (1940). Brit. Heart J., 2, 271.

White, P. D., Chamberlain, F. L., and Graybiel, A. (1941). Brit. Heart J., 3, 233.

Zuckerman, S., Krohn, P. L., Whitteridge, D. (1942). Lancet, 1, 252. 